Document downloaded from:

http://hdl.handle.net/10251/160982

This paper must be cited as:

Gisbert, CM.; Lozano-Galant, JA.; Paya-Zaforteza, I.; Turmo, J. (2020). Calibration of the descent local search algorithm parameters using orthogonal arrays. Computer-Aided Civil and Infrastructure Engineering. 35(9):997-1008. https://doi.org/10.1111/mice.12545

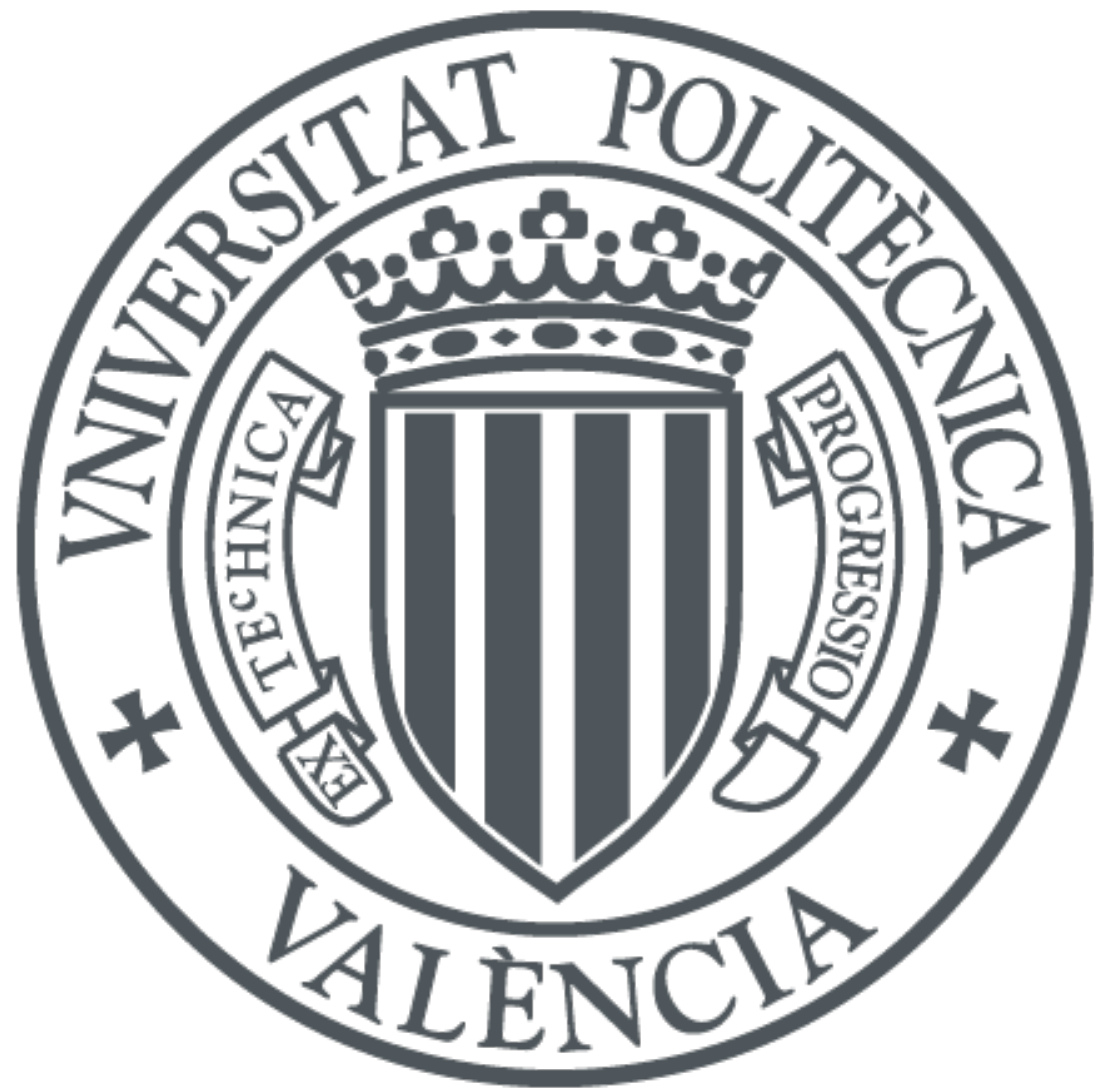

The final publication is available at

https://doi.org/10.1111/mice.12545

Copyright Blackwell Publishing

Additional Information

This is the peer reviewed version of the following article: Gisbert, CM, Lozano-Galant, JA, Paya-Zaforteza, I, Turmo, J. Calibration of the descent local search algorithm parameters using orthogonal arrays. Comput Aided Civ Inf. 2020; 35: 997-1008, which has been published in final form at https://doi.org/10.1111/mice.12545. This article may be used for non-commercial purposes in accordance with Wiley Terms and Conditions for SelfArchiving. 


\title{
Calibration of the Descent Local Search algorithm parameters using orthogonal arrays
}

\author{
Gisbert, C., \\ ETSICCP Universidad Politécnica de Valencia, Camí de Vera, s/n, 46022 Valencia (Spain) \\ \& \\ Lozano-Galant, J.A., \\ ETSICCP Universidad de Castilla-La Mancha Av. Camilo José Cela, s/n 13071 - Ciudad Real (Spain) \\ $\&$ \\ Paya-Zaforteza, I., \\ ETSICCP Universidad Politécnica de Valencia, Camí de Vera, s/n, 46022 Valencia (Spain) \\ $\&$ \\ Turmo, J. \\ ETSICCP Universitat Politècnica de Catalunya BacerlonaTECH. Carrer Jordi Girona, 1, 08034 Barcelona (Spain)
}

\begin{abstract}
Solving optimization problems using heuristic algorithms requires the selection of its parameters. Traditionally, these parameters are selected by a trial and error process that cannot guarantee the quality of the results obtained because not all the potential combinations of parameters are checked. To fill this gap, this paper proposes the application of Taguchi's orthogonal arrays to calibrate the parameters of a heuristic optimization algorithm (the Descent Local Search algorithm). This process is based on the study of the combinations of discrete values of the heuristic tool parameters and it enables to optimize the heuristic tool performance with a reduced computational effort. To check its efficiency, this methodology is applied to a technical challenge never studied before: the optimization of the tensioning process of cable-stayed bridges. The statistical improvement of the heuristic tool performance is studied by the optimization of the tensioning process of a real cable-stayed bridge. Results show that the proposed calibration technique provided robust values of the objective function (with lower minimum and mean values, and lower standard deviation) with reduced computational cost.
\end{abstract}

\section{INTRODUCTION}

Structural optimization using heuristic methods has a major drawback, as the optimized solution depends, to a greater or lesser extent, on the heuristic parameters. Traditionally, several trial and error combinations of these parameters are analyzed to define what parameters fit the studied problem best (see [1-2]). Unfortunately, this approach has two problems: 1) it consumes a lot of time, 2) the quality of the results obtained cannot be guaranteed because not all the potential combinations of parameters can be checked. To fill this gap, this paper proposes the application of Taguchi's orthogonal arrays to calibrate the parameters of a heuristic optimization tool (the Descent Local Search algorithm). This process is based on the study of the combinations of discrete values of the heuristic tool parameters and it enables to optimize the heuristic tool performance. To check its efficiency, this methodology is applied to a technical challenge never studied before: the optimization of the tensioning process of cable-stayed bridges built on temporary supports. The resulting methodology guarantees the achievement of the optimal performance of the optimization tool with reduced computational efforts. To the best of the authors' knowledge, this is a major novelty as it proposes the first application in the literature of the orthogonal arrays for the calibration of heuristic tool parameters. This methodology is characterized by its generality as it can be applied to other heuristic tools and optimization problems.

This paper is organized into the following sections: In Section 2, a literature review of the modelling of the tensioning process of cable-stayed bridges built on temporary supports is reviewed. The main assumptions of the selected simulation algorithm are also presented. In Section 3, the general problem of the optimization of the tensioning process of cable-stayed bridges built on temporary supports is defined. In Section 4 , the design of statistical experiments to calibrate the heuristic tool parameters is presented. In Section 5, the parameters of the heuristic tool are calibrated in a real cable-stayed bridge. 
The superior performance of the calibrated heuristic tool is also showed. Finally, some conclusions are drawn in Section 6.

\section{MODELING THE TENSIONING PROCESS OF CABLE-STAYED BRIDGES}

Cable-stayed bridges stand as one of the most aesthetic man-built structures. Since the first examples of this typology [3], the development of materials, construction techniques and simulation methods, as well as economic well-being, have contributed to a huge proliferation of this kind of bridges.

In cable-stayed bridges, all the resistant mechanisms are related to some extent to the tensile forces of the stay cables and, one of the major challenges of these structures is the materialization of their construction process to ensure the achievement of a target state of stresses and /or deformations in service. This state is known as the Objective Service Stage, OSS [4]. Since modern cablestayed bridges are highly indeterminate systems, there is not just one methodology for calculating the cable stay forces in the OSS.

To achieve the OSS, cable-stayed bridges are traditionally erected either by the cantilever or by the temporary support erection technique $[5,6]$. On the one hand, the cantilever erection method consists of the balanced placement of deck segments supported by stay cables from the pylons. This technique is the most common construction procedure for long span structures as well as for those in locations where no temporary supports can be placed (e.g. on water or soil with low bearing capacity). In the alternative method, the superstructure is first erected using conventional construction techniques on a set of temporary supports. Then, when the stay cables are installed and pre-stressed, the temporary supports are successively lifted, transferring their load to the stay cablesystem.

The OSS can be characterized by a set of stay cable forces. Some of the main criteria used to define these forces in the OSS are summarized in [4]. Among these methods, the Rigidly Continuous Beam Criterion is noteworthy [7]. In this method the stay forces are defined by projecting, into the stays' direction, the vertical reactions of an equivalent continuous beam (in which the anchorages of the stay cables at the deck are replaced by fictitious supports).

Once the target behaviour in the OSS has been defined, they can be clustered into a vector $\left\{\mathrm{N}_{\mathrm{OSS}}\right\}$. This vector includes the target stay forces for the inner cables and the target deformation for the backstays. The elements of $\left\{\mathrm{N}_{\mathrm{OSS}}\right\}$ can be obtained by the sum of a passive $\left\{\mathrm{N}_{\mathrm{P}}\right\}$ and an active $\left\{\mathrm{N}_{\mathrm{A}}\right\}$ state as presented in Equation 1. The former vector includes the passive response produced when a Target Load, TL, is applied to the structure, while the latter one includes the effect of the active prestressing on the stays.

$$
\left\{N_{\text {OSS }}\right\}=\left\{N_{P}\right\}+\left\{N_{A}\right\}=\left\{N_{P}\right\}+[I M]\left\{\varepsilon_{\text {OSS }}\right\}
$$

$\left\{\mathrm{N}_{\mathrm{A}}\right\}$ can be simulated by multiplying a set of imposed strains in the stays $\left\{\varepsilon_{O S S}\right\}$ to an Influence Matrix [IM] that includes the effect of the prestressing in each stay of the structure. The only unknown vector of Equation 1 is $\left\{\varepsilon_{0}\right.$ oss $\}$ and it can be directly defined as follows:

$$
\left\{\varepsilon_{\text {OSS }}\right\}=[I M]^{-1}\left(\left\{N_{\text {OSS }}\right\}-\left\{N_{P}\right\}\right)
$$

in which $[I M]^{-1}$ is the inverse of $[I M]$.

$\left\{\varepsilon_{0 S s}\right\}$ can be used to simulate the construction process of a cable-stayed bridge from a direct approach. In fact, this hypothesis is followed by the Direct Algorithm (DA) to avoid the need for an overall iterative process to assure the achievement of the OSS, significantly reducing the computation time.

\section{- Simulation algorithm}

The definition of an adequate construction procedure that assures the achievement of the OSS on site is a complex nonlinear problem as during construction the structural system does not remain constant and partial structures arise. These partial structures might be more flexible than the completed bridge and might be subjected to higher loads during construction. In fact, many researchers and practitioners have stated the importance of simulating the construction process of cable-stayed bridges [8].

The traditional method to simulate the construction process of a cable-stayed bridge is to start at the OSS and dismantle the structure (according to the opposite construction sequence on site). Several authors have proposed methods based on this "backward" approach both for the temporary supports (Backward Algorithm, BA [9]), and the cantilever erection method [10]). The main inconvenience of this simulation is the difficulty to model any modification in the bridge design and/or in its tensioning strategy. To overcome these problems, a forward simulation, which follows the actual erection sequence on site was proposed for the temporary supports (Forward Algorithm, FA [11]) and the cantilever method [10].

Most of the simulation methods presented in the literature assume that any construction stage can be obtained by activating or deactivating a group of elements, loads or boundary conditions from the following or the preceding construction stages. This hypothesis assumes that the construction process can be simulated by linear superposition of stages. An alternative approach (Direct Algorithm, DA, [12]) proposed a direct simulation of the temporary support erection method avoiding the superposition of stages principle and increasing computational efficiency. To enable the simulation of the time dependent phenomena the direct approach was combined with the forward superposition of stages in the Forward- Direct Algorithm [13]. 
In this work the DA is used to simulate the tensioning process of cable-stayed bridges. The main hypotheses of this algorithm are as follows: (1) Each construction stage is simulated with an independent Finite Element Model (FEM). (2) A Local Iterative Process is used to simulate the lifting of the temporary supports during the tensioning operations. In this process the structural system of the FEM of each construction stage is successively updated (by intruding the new stay cables and removing the temporary supports when they are lifted). In this process, vertical reactions at the temporary supports are evaluated. When tensile stresses are obtained in the temporary supports, they are removed for the subsequent tensioning operations. (3) Once the deck is raised from a temporary support, this is removed. (4) In the FEMs, stay forces are simulated by imposed strains, $\varepsilon$, on the stays. (5) To avoid the requirement of an overall iterative process, the prestressing of the last tensioning operation is based on the unstressed length concept and therefore, the stay cable forces are simulated with the vector $\left\{\varepsilon_{0 s s}\right\}$ defined in Equation (2). A detailed explanation of the Direct Algorithm can be found in [12].

\section{- Optimization of the tensioning process}

A number of scholars have proposed the optimization of the design [14], the stay cable forces [15-18], and the construction planning of the cantilever erection method [19]. Nevertheless, to the best of the authors' knowledge no work addressing the optimization of the tensioning process of cables-stayed bridges built on temporary supports has been found in the literature. Traditionally, this sequence is defined according to the designer's experience on a trial and error basis in two tensioning operations (see for example, the tensioning process of the Talavera de la Reina Bridge [20]). In the first tensioning operation, the stays are introduced in alternative sides from the pylon to the bridge abutments. Each stay is prestressed to nearly $80 \%$ of the cable force in the OSS. Finally, in the second operation, the stay forces are prestressed again to ensure the achievement of the OSS. To fill this gap, the calibration methodology proposed in this paper has been applied to a novel problem: the optimization of the tensioning process of cable-stayed bridges built on temporary supports.

\section{OPTIMIZATION OF THE TENSIONING PROCESS OF CABLE STAYED BRIDGES}

According to Stork et al. [21] optimization techniques can be classified into the following three groups: (1) Exact optimization methods, (2) Surrogate-based optimization methods, and (3) Heuristic and Metaheuristic optimization methods. Among these tools it is important to highlight the Heuristic Algorithms, as they provide adequate solutions for realistic and complex optimization problems [22-34].
For this reason, a heuristic algorithm (Descent Local Search DLS, with a first improvement strategy to explore the solution space) was selected to optimize the tensioning process of cable-stayed bridges in this paper. The DLS has been widely applied to other structural optimization problems (see e.g. $[2,35])$ and starts with an initial feasible solution $\mathrm{So}_{1}$ obtained by giving values randomly to the design variables. This initial solution is transformed into a new solution $\mathrm{So}_{2}$ by slightly changing the values of some of its defining design variables. If $\mathrm{So}_{2}$ is feasible and performs better than $\mathrm{So}_{1}$ in terms of the objective function considered, it is adopted as the new current solution of the optimization problem. Otherwise, $\mathrm{So}_{2}$ is rejected and a new solution is created and checked. The process ends when no improvement of the current solution happens after a predefined number of iterations.

The problem of the optimization of a structure can be expressed as minimizing a certain objective function, with respect to some design variables and subjecting it to some parameters and constraints.

For each value of the design variables, the objective function has a distinct value and defines a point in the solution space.

In this section the Objective functions, Parameters, design variables, and Constraints of the optimization problem are described. The assumed parameters of the DLS algorithm are also analyzed.

\section{- Objective function}

The optimization was based on a unique objective function: minimizing bending moments at the pylon base throughout the construction process. The selection of this function was based on the authors' experience as structural engineers of cable-stayed bridges. In fact, in the temporary support erection method, the structural system of the pylon changes significantly between construction and service stages. During construction, the pylon works in the plane of the bridge as a cantilever subjected to the unbalanced loads of the cables, while in service it is a cantilever stiffened by the whole cable-stayed system. Obviously, the proposed methodology is also valid for any other objective function.

\section{Parameters}

The parameters refer to the fixed characteristics of the FEM of the cable-stayed bridge to be optimized. These features can be divided into the following groups: (1) Geometry: Defined by the node's coordinates and their connectivity in elements, (2) Sections and materials in the pylon, deck and stay cables, and (3) Construction and Service loads (excluding the tensioning operations included in the design variables section). To minimize the computational cost the model simplicity is advised. In this paper, the different bridge elements (pylon, deck and stay 
cables) are simulated with beam elements. It is worth highlighting that all the characteristics of the FEM remain constant throughout the optimization process.

\section{- Design variables}

The design variables are successively updated throughout the optimization process. The following design variables are considered for the optimization of the tensioning process of a cable-stayed bridge: (1) Number of tensioning operations $\mathrm{N}_{\mathrm{op}}$ : This variable defines the number of times the stay cables are tensioned. (2) Tensioning matrix [T]: This matrix defines the tensioning process based on the assumptions of the Direct Algorithm [12]. According to this method, the tensioning operations are simulated by percentages of the stay cable strains in the OSS, $\varepsilon_{\text {oss. }}$ In this way, [T] includes $\mathrm{N}_{\mathrm{op}}$ rows and 2 columns. The first column identifies the cable tensioning order, while the second one defines the percentage of $\varepsilon_{\text {oss }}$ introduced by the tensioning operation. The optimization algorithms must obtain the tensioning matrix $[\mathrm{T}]$ for an optimum value of the targeted objective function. To do so, they start from an initial solution of the tensioning matrix $\left[\mathrm{T}_{\mathrm{i}}\right]$ that is successively updated to get a better value of the objective function. Figure 1 shows a stressing matrix of an academic example of the 4-cable stayed bridge model included in this figure. This structure is tensioned in 8 stages. In the first tensioning operation the four stays are installed and prestressed to $80 \%$ of the $\varepsilon_{\text {oss. }}$ Finally, in the second tensioning operation the four stays are prestressed again to reach $100 \%$ of the $\varepsilon_{\text {oss }}$. This figure also includes the matrix $\left[T_{i+1}\right]$ to illustrate how $[\mathrm{T}]$ is updated throughout the optimization process. This matrix includes random changes (highlighted in bold) in both the tensioning order and the tensioning strains.

\section{- Constraints}

The constraints define the conditions that must be satisfied by the optimization algorithm. The constraints assumed in the analyses are as follows: (1) Ultimate Limit State ULS: The internal forces in the different structural elements, pylon, deck and cables, must not exceed the strength of their materials in ULS during the tensioning process. (2) Cables: No compression forces are allowed in the stay cables in any construction stage. (3) Service Limit State SLS: The nodal displacements must not exceed the maximum deflections allowed in the SLS. Cracking may be allowed to some extent, but it must always be controlled. (4) Tensioning matrix [T]: The definition of the tensioning matrix is limited to two prestressing operations and must satisfy the following conditions: (a) Cable installation: To achieve the bridge geometry, every stay cable must be tensioned at least once. In addition, cables must be installed with a minimal tension stress to compensate for wedge slips. (b) Tensioning strains: To ensure the achievement of the OSS in service, the last tensioning operation of every stay cable must correspond with its $\varepsilon_{\text {oss. }}$ (c) Temporary supports: The temporary supports are lifted when a negative reaction appears at a temporary support, and this element is removed henceforth. (d) Prestressing sequence: The same cable cannot be tensioned in two consecutive tensioning operations. Depending on the satisfied restraints, the matrix [T] can be classified as "without consecutive tensioning operations in one stay" (satisfying d) and "with consecutive tensioning operations in one stay" (not satisfying d). It is important to highlight that is necessary to enable the algorithms to generate the second type of matrices to be able to optimize the number of tensioning operations.

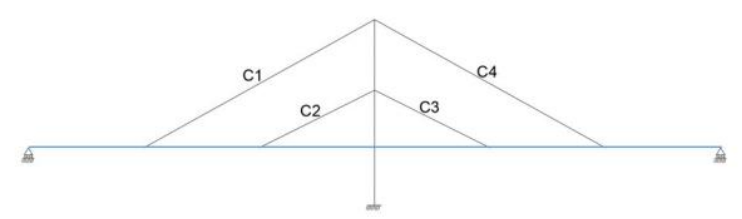

$$
\left[T_{i}\right]=\left[\begin{array}{cc}
3 & 80 \% \\
2 & 80 \% \\
4 & 80 \% \\
1 & 80 \% \\
2 & 100 \% \\
3 & 100 \% \\
1 & 100 \% \\
4 & 100 \%
\end{array}\right] \rightarrow\left[T_{i+1}\right]=\left[\begin{array}{cc}
3 & 80 \% \\
4 & 80 \% \\
2 & 80 \% \\
1 & \mathbf{9 3} \% \\
2 & 100 \% \\
3 & 100 \% \\
1 & 100 \% \\
4 & 100 \%
\end{array}\right]
$$

Figure 1: Examples of tensioning matrices $\left[T_{i}\right]$ and $\left[T_{i+1}\right]$. of a four stays cable-stayed bridge throughout the optimization process.

\section{- Parameters of the heuristic algorithms}

The application of the DLS algorithm requires the common definition of: (1) The maximum number of random variations in the tensioning order. This parameter defines the maximum number of times that the tensioning order of [T] must be randomly modified in each iteration of the optimization algorithm; that is to say, changes in the first column of $[\mathrm{T}]$. In the simulated structure in this paper, three different levels (1,3 and 6) are considered. (2) The maximum number of random variations in the tensioning strains. This characteristic refers to the maximum number of times the tensioning strains are modified in each iteration; that is to say, changes in the second column of $[T]$. Three different levels $(1,2$ and 3$)$ are considered in this paper. (3) The maximum distance between stay cables when tensioned successively. This parameter limits the changes in the tensioning order (first column of [T]) as they are restricted only to those cables located close enough to the one being prestressed. To ease the mathematical representation of this requirement the stays are named in terms of their distance from the tensioned cable as presented in Figure 2. In this figure, the tensioned cable (highlighted with a dotted line) is named 0 and the rest of the stay cables are named successively according to their 
proximity to the stressed cable. For example, Figure 2.a shows how the first stay cable is the one tensioned, and the remaining cables are named 1, 2 and 3 respectively. The numbering of the distance to the second cable when prestressed is presented in Figure 2.b. In this case, three levels of distance are considered (1,2 and 3). (4) The maximum range of variation in the tensioning strains. This parameter refers to the percentage of $\varepsilon_{\text {oss }}$ defined in the second column of $[\mathrm{T}]$. For example, if a cable is tensioned to $80 \%$ of its $\varepsilon_{\text {oss }}$, and the maximum allowed variation of this strain is fixed at $10 \%$, the tensioning strain of the cable in the following iteration would be any random number between $80 \%$ and $90 \%$. In this work, three levels of distance $(10 \%, 50 \%$ and $80 \%)$ are considered in the simulations presented in this work. (5) The type of matrix [T]. These matrices have been defined with two levels (with, W, or without, W/O, consecutive tensioning operations in a stay cable). (6) The maximum number of iterations. To achieve the optimal solution, it is necessary to establish a stopping criterion for the iterative upgrade of the solution. After analyzing the convergence of different examples of growing complexity, this criterion has been fixed by the maximum number of iterations (10000). The parameters required for the simulation of the DLS optimization algorithm are summarized in Table 1.

(a)

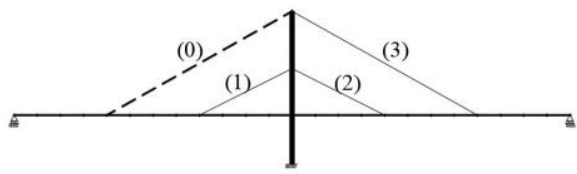

(b)

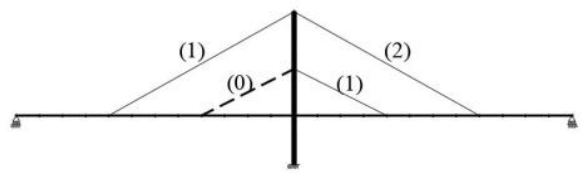

Figure 2: Definition of the distance between the tensioned (represented with a dotted line) and the passive stays cables in two tensioning operations: (a) First cable tensioned, (b) Second cable tensioned.

Table 1. Parameters governing the DLS algorithm

\begin{tabular}{|c|c|}
\hline Parameters & $\mathbf{N}^{\circ}$ of Levels / [Levels] \\
\hline $\begin{array}{l}\text { Maximum number of changes } \\
\text { in the tensioning order }\end{array}$ & $3 /[1,3,6]$ \\
\hline $\begin{array}{l}\text { Maximum number of changes } \\
\text { in the tensioning stresses }\end{array}$ & $3 /[1,2,3]$ \\
\hline $\begin{array}{l}\text { Maximum distance between } \\
\text { tensioned stays }\end{array}$ & $3 /[1,2,3]$ \\
\hline $\begin{array}{l}\text { Maximum change of the } \\
\text { tensioning stress }\end{array}$ & $3 /[10 \%, 50 \%, 80 \%]$ \\
\hline Matrix type & $\begin{array}{c}2 \text { / [With or without consecutive } \\
\text { tensioning operations in a stay } \\
\text { cable] }\end{array}$ \\
\hline $\begin{array}{l}\text { Installation force of the stay } \\
\text { cables }\end{array}$ & 1 / Fixed (constraints) \\
\hline Max number of iterations & $1 /$ Fixed (10000) \\
\hline
\end{tabular}

In the heuristic algorithms, the solution depends, to a greater or lesser extent, on the algorithm parameters. The number of possible combinations of these parameters increases exponentially with the number of levels analyzed. This might lead to an overwhelming number of possible tensioning matrices. This is especially problematic when, as in the case of the heuristic algorithms, many iterations are required to optimize the solution of each of these processes. To solve this problem, trial and error combinations of the algorithm parameters are traditionally analyzed to define the values of the algorithm parameters. Unfortunately, this unsystematised procedure cannot guarantee that the optimal combination of the heuristic algorithm parameters has been analyzed. To fill this gap, a systematic procedure to define the level of the heuristic DLS algorithm parameters based on the design of experiments is proposed in the following section (or, in other words, to calibrate the heuristic tool parameters).

A key characteristic of the proposed methodology is its applicability to heuristic parameters with any number of levels. Obviously, using a higher number of levels (or more allowable parameters) might lead to better optimization results. Nevertheless, to avoid excessive computation costs previous knowledge of the optimization problem is advised to define adequately the parameter levels.

\section{DESIGN OF EXPERIMENTS FOR THE CALIBRATION OF THE DLS PARAMETERS}

The Design of Experiments (DOE) [36] theory is traditionally used to minimize the number of simulations required to draw statistically significant conclusions. This systematic method enables the determination of the relationship between factors affecting a process and the output of that process. In its simplest form, an experiment aims at predicting the outcome by introducing a change of the preconditions represented by one or more independent input variables. The change in one or more of these input variables is generally hypothesized to result in a change in one or more output dependent variables.

The main idea in the DOE refers to the orthogonality between the effects of the studied variables (or factors). In this way, the subset of analyzed experiments is chosen to exploit the information about the most important features of the studied problem, while using a fraction of the effort for full factorial design in terms of experimental runs and resources. In other words, it makes use of the fact that many experiments in full factorial design are often redundant, giving little or no new information about the system.

The advantage of orthogonal arrays is that they provide balanced designs, so factor levels are properly weighted. To illustrate this concept, Figure 3 is presented. The design described in this figure consists of the combination of three variables, A, B and C, with two levels (+) or (-). The aim of this example is to obtain the levels of the variables that 
minimize a certain objective function. On the one hand, Figure 3.a represents a full factorial plan design where all the possible combinations of the variables are analyzed. Each highlighted point of the figure represents a studied experiment. For example, the lowest vertex on the left represents an experiment with the following levels in the variables: A (-), B (-) and C (-). The number of simulations for the full factorial design would be the number of levels to the power of the number of variables; that is $2^{3}=8$. These eight points are depicted as the vertices of the cube in this figure. On the other hand, Figure 3.b shows an orthogonal design defined with the Taguchi's L4 array. This array enables the study of 4 properly selected cases instead of 8 . This figure shows that the projections of all the bases allow the correct identification of the effect of the level changes of each of the variables (in fact, the projection of the cube on all the planes is indeed always a square) [37]. Finally, Figure 3.c shows the analysis of a non-orthogonal experiment, where 4 experiments were randomly selected. The analysis of this case is not very representative as the projections of the cube in the different bases do not provide squares but triangles Unlike the preceding examples, this indicates that the selected experiments might not provide information about the whole system of solutions [37].

Figure 3 was presented to illustrate the importance of the values of the variables in the experiment. As stated in Box et al. [37] the complete projections of the Taguchi's arrays are orthogonal and therefore, they provide the essential information required to characterize the solution space with the minimal number of experiments.

In order to introduce non-experienced engineers and practitioners to the design of orthogonal experiments, in the 60's, Genichi Taguchi [38] developed a set of tables designed to define the minimal set of experiments that provides statistically significant information about the problem. In engineering, this technique has been widely applied (see for example [39-43]).

In the Taguchi tables, known as the orthogonal arrays, the number of experiments depends on the number of variables and their levels. For example, an orthogonal array $\mathrm{L}_{18}$ [36] enables the study of one factor with two levels (factor A) and seven factors with three levels (factors B, C, D, E, F, G and H). (a)

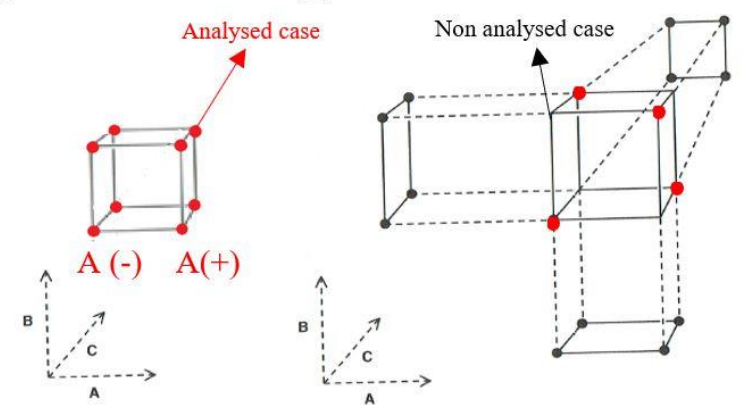

(c)

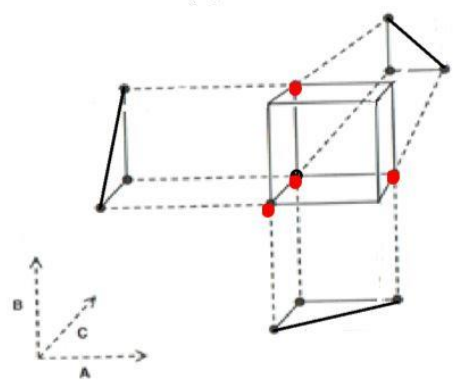

Figure 3. Graphical representation of a (a) Full factorial plan design, (b) Fraction orthogonal plan design with the application of the L4 array, and (c) Fraction non orthogonal plan design with random cases. Adapted from [37].

The number 18 represents the number of experiments required to assure an orthogonal experiment. Each of these experiments is defined in a different row in which the levels of the different factors are detailed. The computational advantages of the orthogonal arrays are obvious as to obtain the same results from a full factorial analysis, a total number of $2 \cdot 7^{3}=1458$ experiments would be required instead of the 18 proposed by this technique.

The levels of the parameters of the analyzed algorithms described in Table 1 leads to 162 DLS experiments for full factorial designs. To minimize the number of experiments required to define the levels of the parameters of the DLS algorithm, the orthogonal arrays are applied in the following section.

\section{- Application of the orthogonal arrays}

The $\mathrm{L}_{18}$ array can be adapted to deal with the only 5 parameters considered (named A, B, C, D and E). In this case, parameters $\mathrm{F}, \mathrm{G}$ and $\mathrm{H}$ will take null values. It is important to highlight that in this procedure it is traditionally advised to assume some columns as null to increase the probability of achieving a successful statistical analysis. The particularization of the L18 array for the analysis of the five factors of the LDS algorithm and their corresponding values is presented in Table 2 .

The levels of these parameters correspond with those presented in Table 1. It is important to highlight that this 
analysis provides enough degrees of freedom to study the residuals (as factors $\mathrm{F}, \mathrm{G}$ and $\mathrm{H}$ are not considered).

In the following section, the DLS algorithm is applied for the optimization of the tensioning process of a cable-stayed bridge. Table 2 shows the considered levels for the factors: (A) Matrix type: 2 levels [with, W, or without, W/O, consecutive tensioning operations in the stay cables]. (B) Maximum number of changes in the tensioning order: 3 levels: $[1,2,6]$. (C) Maximum number of changes in the tensioning stresses: 3 levels $[1,2,3]$. (D) Maximum distance between tensioned stays: 3 levels $[1,2,3]$ and $(E)$ Maximum change of the tensioning stress: 3 levels $[10 \%$, $50 \%, 80 \%]$.

Table 2 Particularization of the $\mathrm{L}_{18}$ array for the analysis of the DLS algorithm.

\begin{tabular}{ccccccccc} 
& \multicolumn{8}{c}{ algorithm. } \\
\cline { 2 - 8 } & A & B & C & D & E & F & G & H \\
\hline $\mathbf{1}$ & W/O & 1 & 1 & 1 & 10 & 0 & 0 & 0 \\
$\mathbf{2}$ & W/O & 1 & 2 & 2 & 50 & 0 & 0 & 0 \\
$\mathbf{3}$ & W/O & 1 & 3 & 3 & 80 & 0 & 0 & 0 \\
$\mathbf{4}$ & W/O & 3 & 1 & 1 & 50 & 0 & 0 & 0 \\
$\mathbf{5}$ & W/O & 3 & 2 & 2 & 80 & 0 & 0 & 0 \\
$\mathbf{6}$ & W/O & 3 & 3 & 3 & 10 & 0 & 0 & 0 \\
$\mathbf{7}$ & W/O & 6 & 1 & 2 & 10 & 0 & 0 & 0 \\
$\mathbf{8}$ & W/O & 6 & 2 & 3 & 50 & 0 & 0 & 0 \\
$\mathbf{9}$ & W/O & 6 & 3 & 1 & 80 & 0 & 0 & 0 \\
$\mathbf{1 0}$ & W & 1 & 1 & 3 & 80 & 0 & 0 & 0 \\
$\mathbf{1 1}$ & W & 1 & 2 & 1 & 10 & 0 & 0 & 0 \\
$\mathbf{1 2}$ & W & 1 & 3 & 2 & 50 & 0 & 0 & 0 \\
$\mathbf{1 3}$ & W & 3 & 1 & 2 & 80 & 0 & 0 & 0 \\
$\mathbf{1 4}$ & W & 3 & 2 & 3 & 10 & 0 & 0 & 0 \\
$\mathbf{1 5}$ & $\mathrm{W}$ & 3 & 3 & 1 & 50 & 0 & 0 & 0 \\
$\mathbf{1 6}$ & W & 6 & 1 & 3 & 50 & 0 & 0 & 0 \\
$\mathbf{1 7}$ & W & 6 & 2 & 1 & 80 & 0 & 0 & 0 \\
$\mathbf{1 8}$ & W & 6 & 3 & 2 & 10 & 0 & 0 & 0 \\
\hline
\end{tabular}

\section{APPLICATION OF THE PROPOSED METHODOLOGY TO A REAL CABLE-STAYED BRIDGE}

In order to show the applicability of the proposed methodology to the tensioning process of a full-scale structure documented in the literature, a simplified model of a cable-stayed bridge for the city of Wuxi in China [12] is analyzed. The model of this structure has a $63 \mathrm{~m}$ high concrete pylon, a $180 \mathrm{~m}$ long steel box girder deck and 18 stays arranged in a semi-harp symmetrical form. The selfweight of the bridge deck, $\mathrm{g}_{1}$, and the target load, TL, are $135 \mathrm{kN} / \mathrm{m}$ and $202.5 \mathrm{kN} / \mathrm{m}$ respectively. The stays are uniformly anchored every $9 \mathrm{~m}$ along the bridge deck and every $1.8 \mathrm{~m}$ along the pylon as presented in Figure 4.a. Alternative examples of cable-stayed bridges can be found in the literature [44-46].

(a)

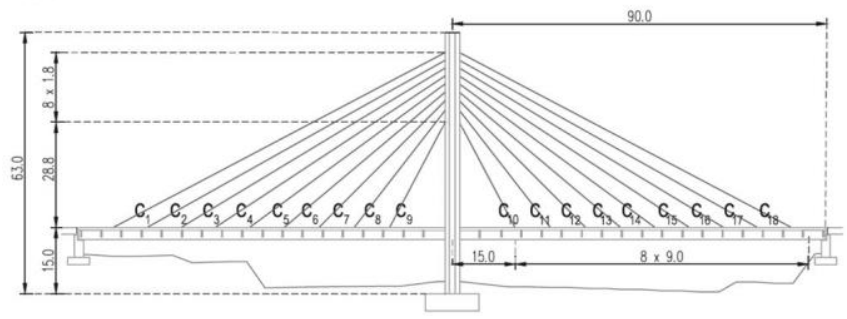

(b)

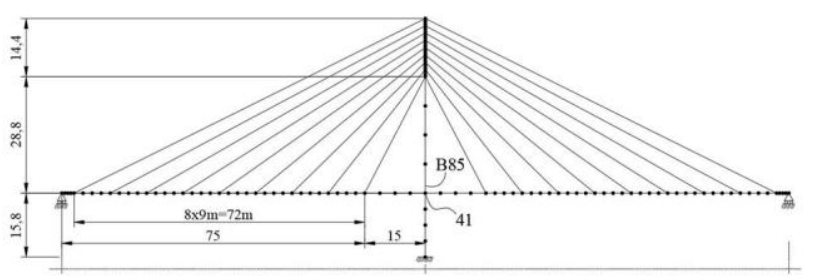

Figure 4. (a) Cable stayed bridge in Wuxi (China). (b) FEM Model. Units in $\mathrm{m}$.

The FEM of this structure is presented in Figure 4.b. This model is composed of 146 beam elements ( 88 for the deck, 40 for the pylon and 18 for the stay cables). The area, inertia and Young modulus of each of these elements is summarized in Table 3.

\begin{tabular}{cccc}
\multicolumn{4}{c}{ Table 3. Mechanical properties of the real structure. } \\
\hline ELEMENT & E (MPa) & $\mathbf{A ~ ( \mathbf { m } ^ { \mathbf { 2 } } )}$ & $\mathbf{I}\left(\mathbf{m}^{\mathbf{4}}\right)$ \\
\hline Deck & 206000 & 1,72 & 4.20 \\
Pylon & 33500 & 8.54 & 14.4 \\
Cables & 195000 & 0.0072 & $0,01 \mathrm{E}-0.9$ \\
\hline
\end{tabular}

The stay cable forces and the $\left\{\mathrm{N}_{\mathrm{OSS}}\right\}$ were calculated to ensure null vertical deflections at the connections between the deck and the stay cables. From Equation (2), the $\left\{\varepsilon_{0}\right.$ ss $\}$ was calculated and the obtained values are presented in Figure 5.a $\left(\left\{\varepsilon_{0 s s}\right\}\right)$ and Figure 5.b ( $\left.\left\{\mathrm{N}_{\mathrm{OSs}}\right\}\right)$ respectively.

The cable-stayed bridge is erected on temporary supports after 36 construction stages. In the initial stage the bridge deck is supported by 18 temporary and 3 permanent supports. The temporary supports are placed below every stay cable. During the simulation of the tensioning process, once a temporary support is raised it is removed from the structure.

The static response of the structure is evaluated assuming geometrical linearity. It is important to take into account that the simulation is not based on a static structural system, but on an evolutive one. In fact, each optimization step is based on the analysis of as many structures as the stressing 
operations described in the tensioning matrix studied. The objective function to be minimized corresponds to the bending moment at the connection of the pylon with the deck (node 41 in beam element 85 as presented in Figure 4). The cable sag is neglected due to the reduced length of the stays.
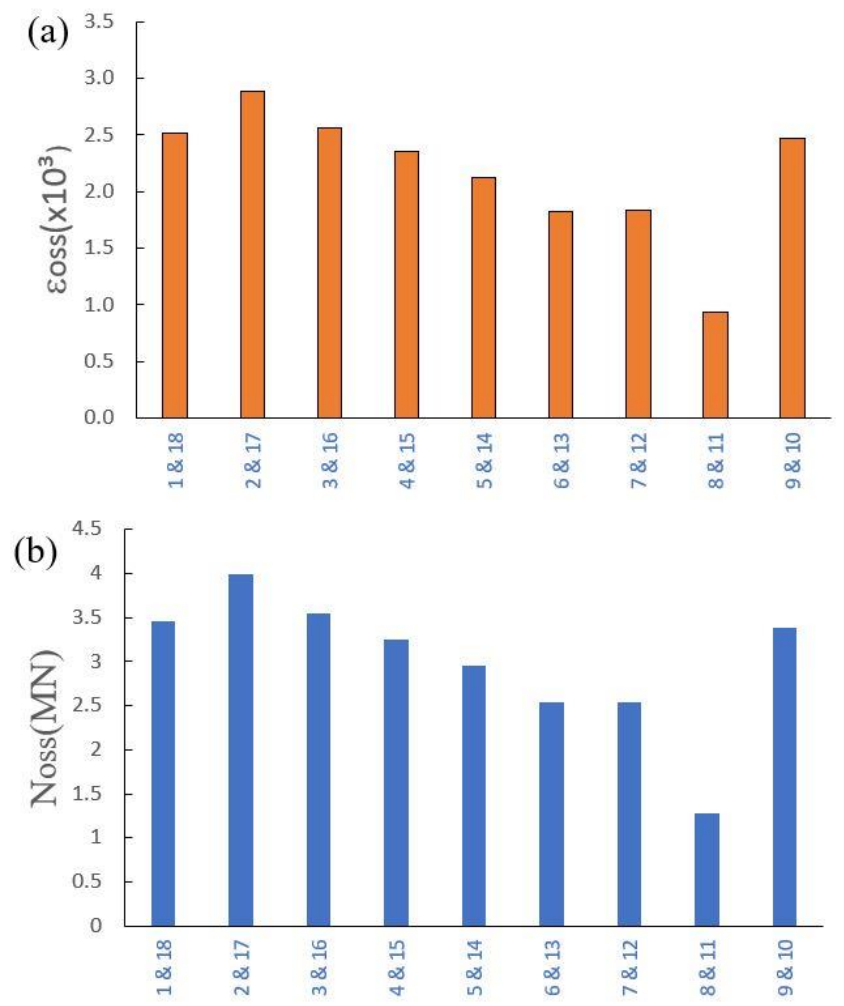

Figure 5: (a) Strains in the OSS, (b) Forces in the stays in the OSS.

The tensioning process of the cable-stayed bridge is optimized with the DLS algorithm. This optimization tool was programmed in Fortran and integrated to the structural analysis code presented in [47-49]. In the following sections, the results of the algorithms are presented and analyzed.

\section{- Analysis of the optimization parameters}

A full factorial analysis of the previous parameters requires 162 tests. Nevertheless, this number can be reduced to 18 when the $L_{18}$ array presented in Table 2 is applied. The use of this array represents a reduction of $88 \%$ of the required tests (from 162 to 18 cases). It is important to highlight that each of these cases requires a significant computational effort as it is based on the optimization of a tensioning process with many construction stages. Concretely, in the analyzed example, 36 different construction stages are required. The first 18 of these stages requires a local iterative process with additional FEM to model the lifting of the temporary supports. The average number of the iterations required in this process was 3 . To assure the convergence of the optimization algorithm based on the previous experience of the authors a maximum of 10.000 iterations were considered and each analysis of the optimization algorithm was repeated 6 times to reduce the randomness of the process. Therefore, reducing from 162 to 18 cases is equivalent to reduce the analysis of a maximum of $(18 \times 3+18) \times 10.000 \times 162 \times 6=699.840 .000$ finite element models to a maximum of $(18 \times 3+18) \times 10.000 \times$ $18 \times 6=77.760 .000$. This can be used to quantify roughly the advantage of the method, even though it is important to highlight that these numbers might $\mathrm{BE}$ altered by the real number of iterations in each simulation.". That way the computation time was reduced from more than 1290 to 140 hours in a computer with an i7 processor and 16GB RAM.

The results of the ANOVA are summarized in Table 4. This table includes the sum of squares, the degrees of freedom (DOF), the $\mathrm{F}$ and the $\mathrm{P}$ values for each factor and their combinations. From these results, the optimal values of the different parameters can be statistically identified by the p-value (see e.g. [37]). This parameter refers to the level of marginal significance within a statistical hypothesis test representing the probability of the occurrence of a given event. A smaller p-value means that there is stronger evidence in favor of the alternative hypothesis. In most analyses, a value of 0.05 is used as the cutoff for significance [38]. In this way, if the p-value is less than 0.05 , we reject the null hypothesis that there is no difference between the means and conclude that a significant difference does exist. As presented in Table 4, this is the case of parameters B (p-value 0.0168) and D (pvalue 0.0024$)$. The rest of the parameters (A, C and E) are not significant.

Table 4: Table summarizing the results of the ANOVA analysis.

\begin{tabular}{ccccc}
\hline Factor & Square sum & DOF & F_Value & P_Value \\
\hline A & $1.93 \mathrm{E}+06$ & 1 & 0.22 & 0.6367 \\
B & $\mathbf{7 . 4 2 E + 0 7}$ & $\mathbf{1}$ & $\mathbf{4 . 3 0}$ & $\mathbf{0 . 0 1 6 8}$ \\
C & $7.50 \mathrm{E}+05$ & 1 & 0.44 & 0.6487 \\
D & $\mathbf{1 . 1 2 E + 0 8}$ & $\mathbf{1}$ & $\mathbf{6 . 5 0}$ & $\mathbf{0 . 0 0 2 4}$ \\
E & $2.33 \mathrm{E}+08$ & 1 & 6.50 & 0.2643 \\
\hline
\end{tabular}

The analysis of the DLS algorithm was based on the 18 optimization runs described in Table 2 . In order to reduce the randomness in each of these simulations, each optimization run was repeated 6 times, leading to a total of 108 analyses. To determine the statistical significance of the different studied factors, an analysis of the variance (ANOVA) of the objective function was performed with the commercial software Statgraphics. In this analysis, the levels of each factor are named 1, 2 and 3 respectively. The results of this analysis are presented in Figure 6. The first part of this figure (Figure 6.a) includes the standard Pareto diagram of the different factors. This diagram shows that the parameters with the highest standardized effects are factor B level 3 and factor D level 3. The second part of the diagram (Figure 6.b) defines the effect of each levels of the five studied factors in the mean standardized value of the 
objective function. This figure shows that the lowest objective functions are obtained for the following factors: A 2, B3, C2, D3 and E2. These levels correspond with the variables defined in Table 5; that is, 6 changes in the tensioning order, 2 changes in the tensioning stress, a maximum distance of 3 between tensioned stays, $10 \%$ maximum change of the tensioning stress and a matrix with consecutive tensioning operations.

The optimal parameters obtained in this section will not remain constant for every structure as they depend on the optimization assumptions and the characteristics of the structure to be optimized. In those structures, another application of Taguchi's Orthogonal arrays will enable the definition of the optimal optimization algorithm parameters.

It is important to highlight two points: 1) the optimal solution was not included in the 18 combinations analyzed, and 2) this combination represents the optimal performance of all 162 possible combinations.

(a)

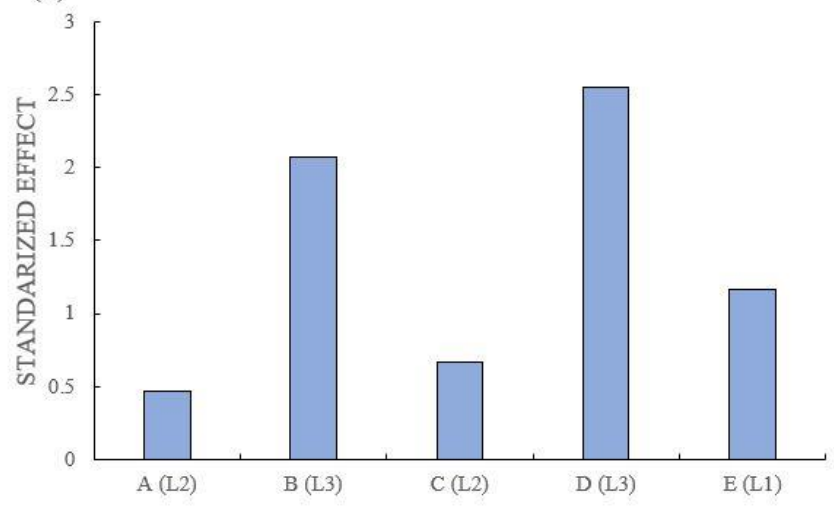

(b)

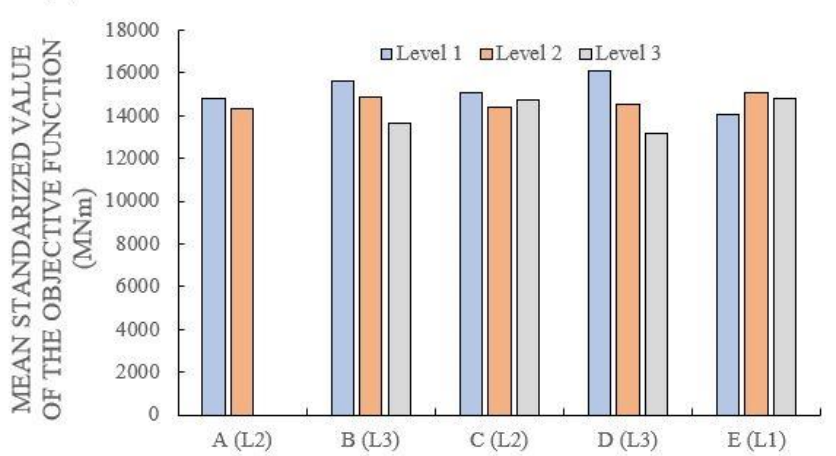

Figure 6: (a) Standardized effect of each factor. (b) Mean standardized value of the objective function.

Table 5. Optimum combination of parameters for the DLS algorithm.

\section{Factor}

A: Matrix Type

B: Maximum changes in the tensioning order

C: Maximum changes in the tensioning stresses

D: Maximum distance between tensioned stays

E: Maximum change of the tensioning stress

\section{- Study of the optimum levels of the DLS}

In order to check the results of the optimum combination of parameters presented in Table 5, a new set of optimization runs (based on this definition of the levels of the parameters) was performed. The obtained results (Test 2 ) were compared with those of the 18 cases introduced into the ANOVA analysis (Test 1). It is important to highlight that each of the tests have been repeated 6 times to reduce the randomness of the solution among different runs. In this way the results correspond to 108 runs (6 repetitions for each of the 18 cases defined in the Table 2) and $6(6$ repetitions for the variables in Table 5) in Tests 1 and 2, respectively.

The statistical analyses of the results of both tests are summarized in Table 6. This table includes the number of simulations, the average, the standard deviation, the minimum and the maximum values of the objective function, the range of the solution (max-min values), the standard bias, and kurtosis coefficients.

Table 6. Summary of the tests.

\begin{tabular}{lcc}
\hline \multicolumn{1}{c}{ Parameter } & Test 1 & Test 2 \\
\hline Number of simulations & 108 & 6 \\
Average objective function $[\mathrm{kNm}]$ & 14774.5 & 10003.3 \\
Standard deviation $[\mathrm{kNm}]$ & 3014.2 & 1380.3 \\
Minimum objective function $[\mathrm{kNm}]$ & 8939.0 & 8500.0 \\
Maximum objective function $[\mathrm{kNm}]$ & 18687.0 & 12878.0 \\
Range $[\mathrm{kNm}]$ & 9748.0 & 4378.0 \\
Standard bias & -1.6 & 1.6 \\
Standard kurtosis & -1.6 & 0.8 \\
\hline
\end{tabular}

The analysis of Table 6 shows that: (1) Lower average objective functions are obtained in Test 2. In fact, the obtained bending moments $(10003.3 \mathrm{kNm})$ are $33.3 \%$ lower than the average in Test 1 (14774.5 kNm). (2) Significantly lower standard deviations are obtained in Test 2, as the obtained value $(1380.3 \mathrm{kNm})$ represents a reduction of $54.2 \%$ of the standard deviation obtained in Test 1 (3014.2 $\mathrm{kNm}$ ). (3) The results of Test 2 results are better bounded as the range of its solutions $(4378.0 \mathrm{kNm})$ is lower than that obtained in Test $1(9748.0 \mathrm{kNm})$. (4) The standard kurtosis of Test $2(0.8)$ is lower than that of Test $1(-1.6)$.

The frequency histogram is presented in Figure 7.a. This figure shows how the results of Test 2 are better bounded than in Test 1. This is also appreciable in Figure 7.b, where the box and whisker plot of each test is presented. The analysis of this graph shows that the lower and upper quartiles are closer in Test 2. In addition, this set has a lower number of outliers and its mean value is smaller.

To check if the factor levels of Test 2 are statistically significant, a statistical hypothesis test was carried out [37]. In this procedure, the statistical relationship between two data sets is compared as an alternative to an idealized null hypothesis, $\mathrm{H}_{0}$, which proposes no relationship between the two data sets. The comparison is deemed statistically significant if the relationship between the data sets would be an unlikely realization of the null hypothesis according 
to a threshold probability (p-value lower than 0.05). The following analyses were carried out: (1) $\mathrm{H}_{0}$ : "The average values of Test 1 and Test 2 are equal". Comparison between average values shows that the confidence interval for Test 1 is $10003.35+/-1061.04 \mathrm{kNm}$, while for Test 2 it is $14774.51+/-847.77 \mathrm{kNm}$. The null hypothesis was rejected after obtaining a P-value lower than 0.05 (specifically 0.00002). (2) $\mathrm{H}_{0}$ : "The standard deviation of Test 1 and Test 2 are equal": Working in a similar way with the standard deviation, confidence ranges of [932.37; 2644.44] and [2522.03; 3746.92] kNm were obtained for Tests 1 and 2 respectively. This null hypothesis is also rejected after obtaining a P-value of 0.024 . These results show that the improvement in both the average values and the standard deviation of Test 2 are statistically significant.

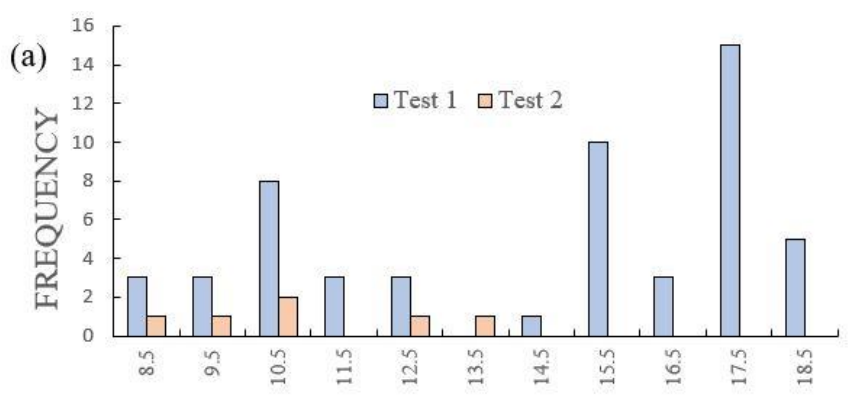

Objective Function (MNm)

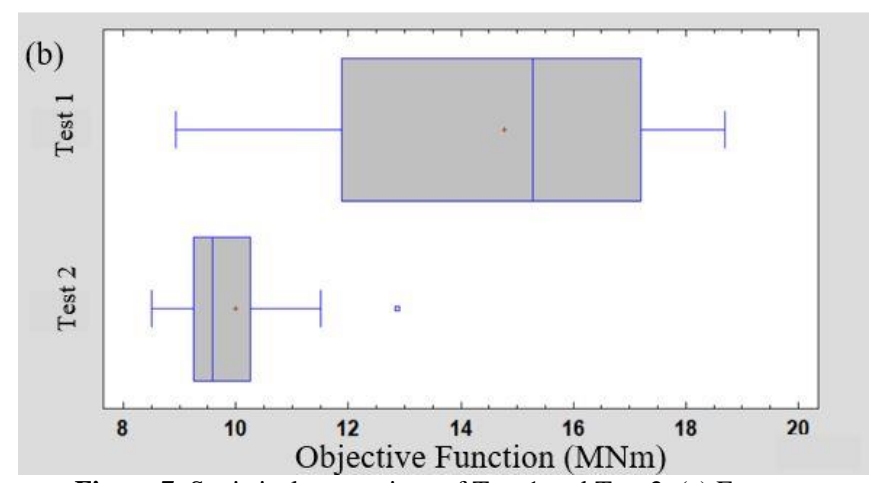

Figure 7: Statistical comparison of Test 1 and Test 2: (a) Frequency Histogram, (b) Box \& Whisker plot.

From all the results presented above, it can be concluded that use of the proposed levels of the variables in the DLS algorithm leads to statistically better results than those obtained with a random or arbitrary selection of those levels.

\section{CONCLUSIONS}

Traditionally the parameters of the heuristic optimization algorithms are selected by a trial and error process. The main inconvenience of this methodology is that it cannot guarantee the quality of the obtained results because not all the potential combinations of parameters are checked. To fill this gap, this paper proposes the application of Taguchi's orthogonal arrays to calibrate the parameters of a heuristic optimization tool (the Descent Local Search algorithm). This methodology is based on the study of the combinations of discrete values of the heuristic algorithm parameters and it enables to optimize the heuristic tool performance with a reduced computational effort. To check its efficiency, this methodology is applied to a technical challenge never studied before: the optimization of the tensioning process of cable-stayed bridges. The advantages of the proposed calibration are illustrated in the example. In fact, the proposed calibration of the heuristic algorithm parameters provided more robust values of the objective function (with lower mean and lower standard deviation) with a significantly lower computational cost.

It is important to highlight the generality of the proposed methodology as it can be applied to other optimization problems and optimization tools. This application will require the following steps: (1) Identifying the variables, constraints and parameters of the problem to be optimized. (2) Identifying the parameters and the levels of the optimization tool. (3) Choosing and adapting an adequate orthogonal array to determine the different combinations of the levels of the optimization tool parameters. (4) Doing an ANOVA analysis to determine the optimal levels of the optimization tool parameters

\section{ACKNOWLEDGMENTS}

The authors are indebted to the Spanish Ministry of Economy and Competitiveness for the funding provided through the research projects BIA2013-47290-R and BIA2017-86811-C2- 1-R founded with FEDER funds and directed by Professor José Turmo and through the research project BIA2017-86811-C2-2-R directed by José Antonio Lozano-Galant.

\section{REFERENCES}

1- García-Segura T., Yepes V., Martí J., Alcalá J. (2014). "Optimization of concrete I-beams using a new hybrid glowworm swarm algorithm. Latin American Journal of Solids and Structures", 11, 1190-1205.

2- Yepes V., Dasí-Gil M., Martínez-Muñoz D., LópezDesfilis V., Martí J. (2019). "Heuristic Techniques for the Design of Steel-Concrete Composite Pedestrian Bridges". Applied Sciences. 3253. 10.3390/app9163253.

3- Lozano-Galant, J.A. \& Payá-Zaforteza, I. (2017) "Analysis of Eduardo Torroja's Tempul Aqueduct an important precursor of modern cable-stayed bridges, extradosed bridges and prestressed concrete", Engineering Structures, 150, 955-968. 
4- Jorquera-Lucerga, J.J. Lozano-Galant, J.A. \& Turmo, J. (2016) "Structural behavior of nonsymmetrical steel cable-stayed bridges", Steel and Composite Structures, 20 (2), 447-468.

5- Behin, Z. \& Murray, D. (1992) "A substructurefrontal technique for cantilever erection analysis of cable-stayed bridges," Comput Struct, 42, 145-57.

6- Gimsing, N. (1997) "Cable supported bridges, Concept and Design", Chichester: John Wiley and Sons.

7- Lazar, B.E. Troitsky, M.S. \& Douglas, M.C. (1972) "Load Analysis Balancing of Cable Stayed Bridges", Structural Division ASCE, 92(8), 17251740.

8- Janjic, D. Pircher, M. \& Pircher, H. (2003), "Optimization of cable tensioning in cable-stayed bridges", J. Bridge Eng., 8(3), 131-137.

9- Lozano-Galant, J.A. Paya-Zaforteza, I. Xu, D. \& Turmo, J. (2012a), "Analysis of the construction process of cable-stayed bridges built on temporary supports", Eng. Struct., 40, 95-106.

10- Wang P.H. Tang T. \& Zheng H. (2004) "Analysis of cable-stayed bridges during construction by cantilever method". Comput Struct, 82, 329-46.

11- Lozano-Galant, J.A. Paya-Zaforteza, I. Xu, D. \& Turmo, J. (2012b), "Forward Algorithm for the construction control of cable-stayed bridges built on temporary supports", Eng. Struct., 40, 119-130.

12- Lozano-Galant, J.A. Xu, D. Paya-Zaforteza, I. \& Turmo, J. (2013), "Direct simulation of the tensioning process of cable-stayed bridges", Comput. Struct., 121, 64-75.

13- Lozano-Galant, J.A. \& Turmo, J. (2014), “An algorithm for simulation of concrete cable-stayed bridges built on temporary supports and considering time dependent effects", Eng. Struct., 79, 341-353.

14- Martins, A.M.B. Simões, L.M.C. \& Negrão, J.H.J.O. (2016) "Optimum design of concrete cable-stayed bridges", Engineering Optimization, 48(5), 772-791.

15- Mohammad, H.A, Zarbaf, S.E. Norouzi, M. Allemang, R.J. Hunt, V.J. \& Helmicki, A. (2017) "Stay Cable Tension Estimation of Cable-Stayed Bridges Using Genetic Algorithm and Particle Swarm Optimization", Journal of Bridge Engineering, 22(10), 05017008.

16- Ye, X. Sun, Z. \& Huang, G. (2017) "Assessment of the current state of internal force and the optimization of cable force for an existing longspan Pre-Stressed Concrete (PC) cable-stayed bridge", Open Civil Engineering Journal, 11, 572585.

17- Fabbrocino, F. Modano, M. Farina, I. Carpentieri, G. \& Fraternali, F. (2017) "Optimal prestress design of composite cable-stayed bridges", Composite Structures, 169, 167-172.

18- Chen, Z.J. Liu, Y. Yang L.F. \& Zhang, S.B. (2016) "Optimization of stay cable tension of completed bridge of single-pylon cable-stayed bridge based on particle swarm optimization algorithm", Bridge Construction, 46(3), 40-44.

19- Sung, Y.C. Wang, C.Y. \& Teo, E.H. (2016) "Application of particle swarm optimisation to construction planning for cable-stayed bridges by the cantilever erection method", Structure and Infrastructure Engineering, 12(2), 208-222.

20- Sánchez de León, R. Bernal, C. \& Sánchez de León, F. (2012) "Puente atirantado de Talavera de la Reina". Hormigón y Acero, 63, 263, 7-47. In Spanish.

21- Stork, K. Eiben, A.E. \& Bartz-Beielstein, T. (2018) "A new Taxonomy of Continuous Global Optimization Algorithms", Technology Art Science, CIplus Band 4/2018

22- Penadés-Plà, V. García-Segura, T., \& Yepes, V. (2019) "Accelerated optimization method for lowembodied energy concrete box-girder bridge design”, Engineering Structures, 179, 556-565.

23- Siddique, N. \& Adeli, H. (2017) "Nature Inspired computing - Physics- and Chemistry-based Algorithms", CRC Press, Taylor \& Francis, Boca Raton, Florida.

24- Siddique, N.H. \& Adeli, H. (2014) "Spiral Dynamics Algorithm," International Journal on Artificial Intelligence Tools s, 23(6), 1430001.

25- Siddique, N.H. \& Adeli, H. (2014) "Water Drop Algorithms," International Journal on Artificial Intelligence Tools, 23(6), 1430002.

26- Siddique, N.H. \& Adeli, H. (2015) "Harmony Search Algorithm and Its Variants," International Journal of Pattern Recognition and Artificial Intelligence, 29(8), 1539001.

27- Siddique, N. \& Adeli, H. (2016) "Simulated Annealing, Its Variants and Engineering Applications", International Journal on Artificial Intelligence Tools, 25(6), 1630001.

28- Siddique, N. \& Adeli, H. (2017) "Nature Inspired Chemical Reaction Optimization Algorithms," Cognitive Computation, 9(4), DOI 10.1007/s12559017-9485-1.

29- Wang, J. Zhong, D. Wang, D. Liu, H. \& Adeli, H. (2018) "Smart Bacteria Foraging Algorithm-Based 
Customized Kernel Support Vector Regression and EPNN for Compaction Quality Assessment and Control of Earth-Rock Dam," Expert Systems, 35(6), DOI: 10.1111/exsy.12357.

30- Imran Hossain, S. Akhand, M.A.H. Shuvo, M.I.R. Siddique, N. \& Adeli, H. (2019) "Optimization of University Course Scheduling Problem using Particle Swarm Optimization with Selective Search," Expert Systems with Applications, 127, 924.

31- Chikahiro, Y. Ario, I. Pawlowski, P. Graczykowski, C. \& Holnicki-Szulc, J. (2019) "Optimization of reinforcement layout of scissor-type bridge using differential evolution algorithm", Computer-Aided Civil and Infrastructure Engineering, 34(6), 523538.

32- Yin, Y. Li, D. Besinovic, N. \& Cao, Z. (2019) "Hybrid Demand-Driven and Cyclic Timetabling Considering Rolling Stock Circulation for a Bidirectional Railway Line", Computer-Aided Civil and Infrastructure Engineering, 34(2), 164-187.

33- Tao, C. Watts, B. Ferraro, C.C. \& Masters, F.J. (2019) "A Multivariate Computational Framework to Characterize and Rate Virtual Portland Cements", Computer-Aided Civil and Infrastructure Engineering, 34(3), 266-278.

34- Xu, M. Ouyang, M. Mao, Z. \& Xu, X. (2019) "Improving repair sequence scheduling methods for postdisaster critical infrastructure systems", Computer-Aided Civil and Infrastructure Engineering, 34(6), 506-522.

35- Carbonell A., Gonzalez-Vidosa F., Yepes V. (2011). "Design of reinforced concrete road vaults by heuristic optimization". Advances in Engineering Software. 42. 151-159.

36- Taguchi, G. Chowdhury, S. \& Wu, Y. (2004) "Taguchi's Quality Engineering Handbook", Wiley.

37- Box G., Hunter, J.S. Hunter, W.G. (2005) "Statistics for Experimenters," New Jersey, Wiley.

38- Taguchi, G. Chowdhury, S. \& Taguchi, S. (1999) "Robust engineering: learn how to boost quality while reducing costs \& time to market", New York, McGraw-Hill.

39- Arıc1, E. \& Keleştemur, O. (2019) "Optimization of mortars containing steel scale using Taguchi based grey relational analysis method", Construction and Building Materials, 214, 232-241.

40- Manjunath, R. Narasimhan, M.C. Umesh, K.M. Shivam K. \& Bala, U.K. (2019) "Studies on development of high performance, self-compacting alkali activated slag concrete mixes using industrial wastes", Construction and Building Materials, 198, 133-147.

41- Cao, Y.F. Tao, Z. Pan, Z. \& Wuhrer, R. (2018) "Effect of calcium aluminate cement on geopolymer concrete cured at ambient temperature" Construction and Building Materials, 191, 242-252.

42- Madihalli, R. Dattatreya, J.K. \& Latha, M.S. (2018) "Optimization of high-performance concrete mix incorporating fly ash and slag aggregates using statistical approach" International Journal of Civil Engineering and Technology, 9(11), 1870-1882.

43- Chao, L.C. \& Kuo, C.P. (2018) "Optimizing the ultimate strength of precast reinforced concrete pipes in three-edge bearing tests", Structural Concrete, 19(4), 1174-1184.

44- Adeli, H. \& Zhang, J. (1995) "Fully Nonlinear Analysis of Composite Girder Cable-Stayed Bridges", Computers and Structures, 54(2), 267277.

45- Kim, H. \& Adeli, H. (2005) "Wavelet Hybrid Feedback-LMS Algorithm for Robust Control of Cable-Stayed Bridges", Journal of Bridge Engineering, ASCE, 10(2), 116-123.

46- Wu, W.H. Chen, C.C. Jhou, J.W. \& Lai, G. (2018), "A rapidly convergent empirical mode decomposition method for analysing the environmental temperature effects on stay cable force," Computer-Aided Civil and Infrastructure Engineering, 33(8), 672-690.

47- Lozano-Galant, J.A. \& Turmo, J. (2014) "Creep and shrinkage effects in service stresses of concrete cable-stayed bridges", Comput. Concrete, 13(4), 483-499.

48- Lozano-Galant, J.A. Paya-Zaforteza, I. \& Turmo, J. (2015) "Effects in service of the staggered construction of cable-stayed bridges built on temporary supports", The Baltic J. Road Bridge Eng., 10(3), 247-254.

49- Lozano-Galant, J.A. Nogal, M. Turmo, J. \& Castillo, E. (2015) "Selection of measurement sets in static structural identification of bridges using observability trees", Computers and Concrete, 15(5), 771-794. 